\title{
Biofilm Formation and its Correlation with ESBL Production in Klebsiella pneumoniae Isolated from a Tertiary Care Hospital
}

\author{
Lathamani K ${ }^{1}$, Subbannayya Kotigadde ${ }^{2}$ \\ ${ }^{1,2}$ Department of Microbiology, KVG Medical College, Sullia
}

\begin{abstract}
Klebsiella pneumoniae, an important biofilm forming organism found to be responsible for a wide range of infections especially when they grow as biofilm...Biofilm formation facilitates their survival in a hostile environment of host immune system and antimicrobials .ESBL producing K.pneumoniae isolates had a greater ability to form biofilm than non biofilm producing isolates. As there is hardly any literature found regarding the correlation between biofilm production and drug resistance in respect to ESBL production by the isolates of K.pneumoniae in Dakshina Kannada District, Karnataka, the present study has been conducted. A total of 347 isolates of K.pneumoniae collected between June 2012 and October 2014 were characterized according to standard microbiological procedures. Detection of biofilm was done by tissue culture plate method. Antimicrobial susceptibility pattern of the bio film producing strains was determined by the standard Kirby Bauer disc diffusion method and presumptive ESBL production by double disk synergy test (DDST). Results: Out of a total of 347 clinical isolates of Klebsiella pneumoniae, 167 isolates (48.13\%)were found to be biofilm producers .Out of a total of 183 resistant strains of isolates of K.pneumoniae screened, 93(50.81\%) were ESBL producers and 70(75.26\%) of 93 ESBL producers were biofilm producers. ESBL producing Klebsiella pneumoniae isolates (75.26\%) had an exceptional capacity to form biofilm, in comparison with non ESBL forming K.pneumoniae(24.74\%).
\end{abstract}

Keywords: Klebsiella pneumoniae, Drug resistance, Bio film, In dwelling medical devices, Tissue culture plate method

\section{Introduction}

Klebsiella pneumonia, an important opportunistic pathogen liable for wide range of hospital acquired and community acquired infections to the patients especially when they are on indwelling devices, on which these organisms grow as a biofilm. ${ }^{4}$ The virulence factors like capsule, lipopolysaccharide and type 1 or type 3 fimbriae are responsible for K.pneumoniae to form biofilm. ${ }^{4}$ Biofilm consists of group of microorganisms embedded in a extracellular polysaccharides matrix, which make them to adherent to biotic and abiotic surfaces in order to escape the activity of defense mechanism of the host, killing effect of antibiotic therapy and also the action of disinfectants. ${ }^{13}$ Klebsiella pneumonia is an important bacteria responsible for the formation of biofilm and cause various infection which make them one of the eight most important nosocomial pathogen.$^{11}$ The raise in the global spread of resistance towards antibiotics mostly in hospital area among K.pneumoniae strains is observed especially when they form biofilm . ${ }^{4}$ The required concentration of antibacterial substances in order to kill the organism may increase the level up to 1000 folds when they grow as biofilm. ${ }^{1}$ Bacterial motility, surface adhesions, availability of key nutrients, chemotaxis towards surface and presence of surfactants are some factors which influence biofim formation. Certain other factors like, temperature, $\mathrm{pH}$ also have been shown to influence biofilm formation by microorganisms in different conditions. ${ }^{1,14}$ According to National Institutes of Health, “ more than $60 \%$ of all microbial infections are caused by biofilms."Although the percentage quoted is high, it seems correlates when considering the infections caused by them such as middle ear infections of children,UTI, dental plaque, catheter infections, gingivitis etc. recur and difficult to treat. $^{6}$ The tissue culture plate method was opted to determine biofilm formation.It is a reliable and quantitative method to detect biofilm formation. ${ }^{1}$ Klebsiella pneumonia, responsible for serious outbreaks of multi drug resistant diseases due to un controlled usage of antibiotics. Various mechanisms of resistance towards antibiotics are enzymatic inactivation of antibiotics, altered target site, alteration of metabolic pathway and decreased porin permeability. ${ }^{18}$ Beta lactam antibiotics show drug resistance mainly due to enzymatic inactivation or alteration of antibiotics. ${ }^{19}$ The enzymatic inactivation of antibiotics in order to show resistance to third generation cephalosporins in case of K.pneumoniae is mainly by the production of extended spectrum beta lactamase enzymes (ESBLs).

Extended spectrum $\beta$-lactamases are beta lactamase enzymes able to hydrolyze the effects of penicillins and broadspectrum cephalosporins. But the hydrolyzing effect is interfered by the presence of beta lactamase inhibitors. One of the phenotypic method of testing of ESBLs is double disk synergy test (DDST). Double disk synergy test is a test which could be adopted in any of the laboratory as a routine diagnostic test due to its low cost and easy management. Studies have shown that there is a liaison between ESBL production and the biofilm formation of $K$. pneumoniae isolates. It has been understood that very few studies have been conducted in order to evaluate the correlation between biofilm formation and ESBL production among K.pneumoniae in Dakshina Kannada District. Since the literature showed the increased occurrence of ESBL production among the strains of K.pneumoniae and also biofilms are more connected to ESBL producers than to non ESBL producers. ${ }^{2,4,6}$ The present study was conducted to know the resistance pattern of K.pneumoniae towards various antibiotics, ESBL production among the resistant strains of K.pneumoniae and to determine the correlation between biofilm formation and ESBL production among the resistant strains of K.pneumoniae. 


\section{International Journal of Science and Research (IJSR) \\ ISSN (Online): 2319-7064}

Index Copernicus Value (2013): 6.14 | Impact Factor (2014): 5.611

\section{Methodology}

Study Population: The proposed study was carried out in the Department of Microbiology, KVG Medical College and hospital, Sullia, Karnataka. A total of 347 isolates collected between June 2012 and October 2014 were studied for biofilm formation and its correlation to anti microbial resistance pattern and ESBL production.

\section{Phenotypic Identification:}

The isolates of K.pneumoniae were identified according to standard microbiological procedures. ${ }^{7}$ Antimicrobial Susceptibility Tests: ${ }^{3,17}$

Antibacterial susceptibility pattern of all isolates of K.pneumoniae were determined by the standard Kirby Bauer disc diffusion method. The Muller-Hinton agar plate was inoculated with a suspension of K.pneumoniae adjusted to 0.5 McFarland turbidity standards, $\left(1 \times 10^{8} \mathrm{cfu} / \mathrm{ml}\right)$. The antimicrobial disks used were ceftazidime $(30 \mu \mathrm{g})$, cefotaxime $(30 \mu \mathrm{g})$, ceftriaxone $(30 \mu \mathrm{g})$, amikacin $(30 \mu \mathrm{g})$, ciprofloxacin $(5 \mu \mathrm{g})$, gentamicin $(10 \mu \mathrm{g})$, meropenem $(10 \mu \mathrm{g})$ and ertapenem $(10 \mu \mathrm{g})$. The plates were incubated overnight at $37^{\circ} \mathrm{C}$. The zones of inhibition were measured and interpreted comparing with the standard measurement chart.

Detection of ESBL production by Doule disk synergy test $^{5}$ :The disk containing Amoxicillin - clavulanate was placed at the centre of the lawn culture made on Muller Hinton Agar (MHA)plate inoculated with each of the isolates of K.pneumoniae found to be resistance towards any one or all the antibiotic disks of ceftazidime, cefotaxime and ceftriaxone. The discs of ceftazidime, cefotaxime and ceftriaxone each having a disc concentration of $30 \mu \mathrm{g}$ were placed around the central amoxicillin - clavulanate disc at a centre to centre distance of $30 \mathrm{~mm}$. The plate was incubated at $37^{\circ} \mathrm{C}$ for $24 \mathrm{hrs}$. If there was any enhancement of zone of inhibition between any one of the cephalosporin disks with the central disk the isolate was considered as ESBL producer.

Biofilm formation: Tissue culture plate method(TCP) was chosen to detect the biofilm formation.

Ten milliliter of trypticase soybroth containing $1 \%$ glucose was taken to inoculate each isolates of K.pneumoniae and incubated at $37^{\circ} \mathrm{C}$ for 24 hours. The growth were then suspended in to fresh medium in 1:100 dilution. Two thousand micro liters of diluted cultures were filled in different polystyrene wells and incubate at $37^{\circ} \mathrm{C}$ for 24 hours. The contents of each well were removed after attaining required time of incubation by gentle tapping. The wells were washed with $0.2 \mathrm{ml}$ of phosphate buffer saline and the wells were fixed by $2 \%$ sodium acetate and stained by $0.1 \%$ crystal violet. The stain was washed and optical density was measured at wavelength of $580 \mathrm{~nm} .^{1}$

$\begin{array}{lll}\text { Interpretation: } & & \\ \text { Mean O D values } & \text { Adherence } & \text { Biofilm formation } \\ <0.120 & \text { None } & \text { None/weak } \\ 0.120-0.240 & \text { Moderate } & \text { Moderate } \\ >0.240 & \text { Strong } & \text { High } \\ \text { 3. Results } & & \end{array}$

\section{Results}

Out of a total of 347 clinical isolates of Klebsiella pneumoniae, 167 isolates $(48.13 \%)$ were found to be biofilm producers and $180(51.87 \%)$ biofilm non producers. A total of 183 resistant strains of isolates of K.pneumoniae screened, 93(50.81\%) were ESBL producers and $70(75.26 \%)$ of $93 \mathrm{ESBL}$ producers were biofilm producers.

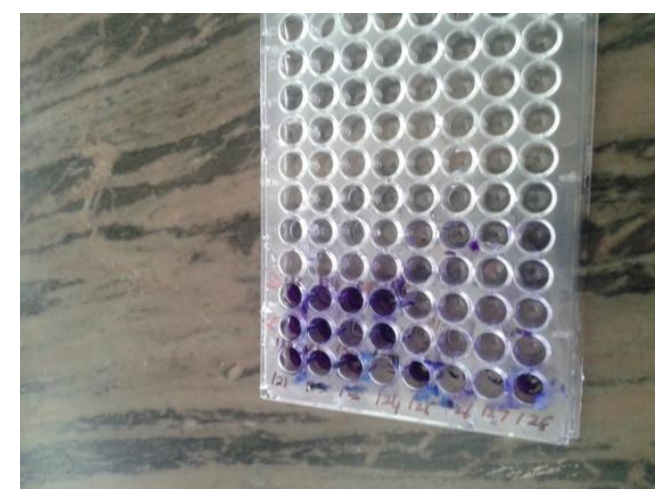

Figure 1: Showing occurrence of biofilm among K.pneumoniae

Table 1: The susceptibility pattern of clinical isolates of

\begin{tabular}{|c|c|c|}
\multicolumn{3}{|c|}{ K.pneumoniae $(\mathrm{n}=347)$} \\
\hline Antibiotics & Susceptibility & Resistance \\
\hline cefotaxime & I82(52.45\%) & $165(47.55 \%)$ \\
\hline ceftazidime & $149(42.94 \%)$ & $198(57.06 \%)$ \\
\hline ceftriaxone & I90(54.75\%) & $157(45.25 \%)$ \\
\hline ciprofloxacin & $199(57.35 \%)$ & $148(42.65 \%)$ \\
\hline gentamicin & $243(70.02 \%)$ & $104(29.98 \%)$ \\
\hline amikacin & $295(85.02 \%)$ & $52(14.98 \%)$ \\
\hline ertapenem & $329(94.81 \%)$ & $18(5.19 \%)$ \\
\hline meropenem & $340(97.98 \%)$ & $7(2.02 \%)$ \\
\hline
\end{tabular}


International Journal of Science and Research (IJSR)

ISSN (Online): 2319-7064

Index Copernicus Value (2013): 6.14 | Impact Factor (2014): 5.611

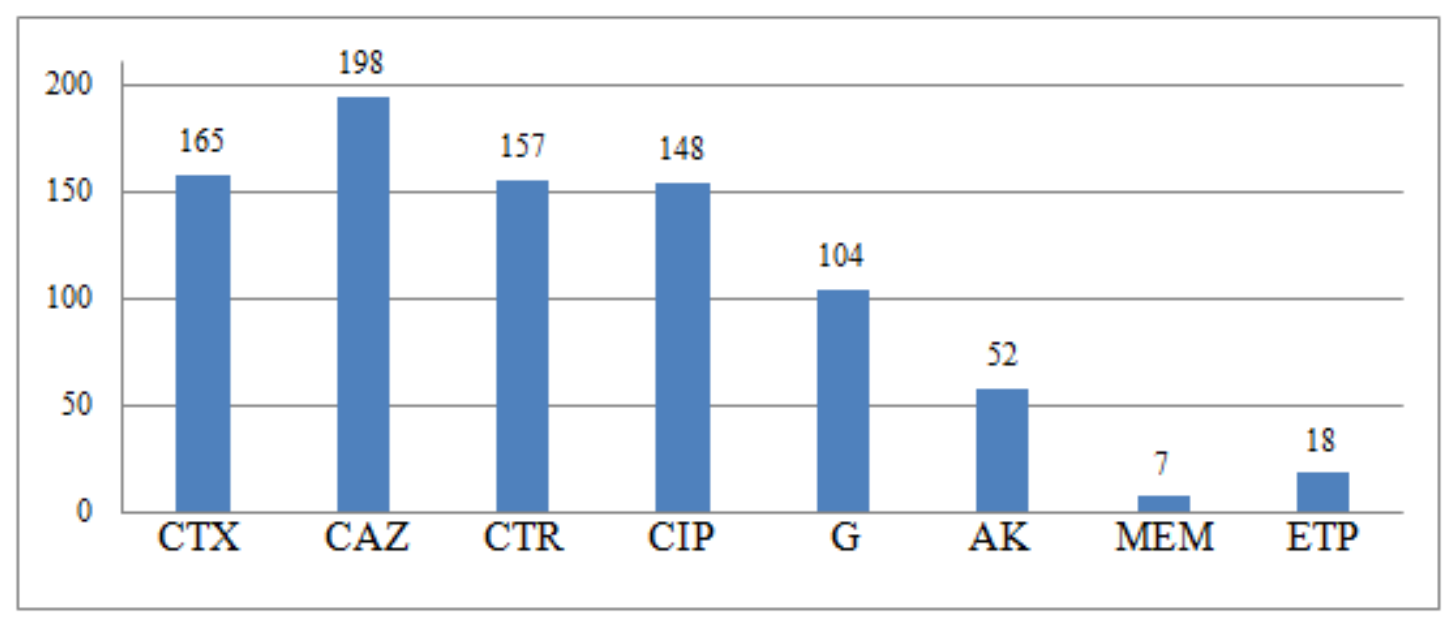

Figure 2: Occurrence of antibiotic resistance among K.pneumoniae

Table 2: Resistance pattern of biofilm positive and negative isolates of K.pneumoniae

\begin{tabular}{|c|c|c|c|}
\hline \multicolumn{4}{|c|}{ Resistance } \\
\hline S. No & Antibiotics & $\begin{array}{c}\text { Biofilm positive } \\
\text { isolates, } \mathrm{n}=198\end{array}$ & $\begin{array}{c}\text { Biofilm negative } \\
\text { isolates n=149 }\end{array}$ \\
\hline 1 & CTX & $114(57.57 \%)$ & $51(34.22 \%)$ \\
\hline 2 & CAZ & $130(65.65 \%)$ & $68(45.63 \%)$ \\
\hline 3 & CTR & $112(56.56 \%)$ & $46(30.87 \%)$ \\
\hline 4 & CIP & $106(53.53 \%)$ & $42(28.18 \%)$ \\
\hline 5 & G & $64(32.32 \%)$ & $42(28.18 \%)$ \\
\hline 6 & AK & $46(23.23 \%)$ & $8(5.36 \%)$ \\
\hline 7 & ETP & $9(4.54 \%)$ & $9(6.04 \%)$ \\
\hline 8 & MEM & $4(2.02 \%)$ & $3(3.14 \%)$ \\
\hline
\end{tabular}

\section{Discussion}

Bacteria producing biofilm are accountable for many non compliant infections and are difficult to destroy and treat. They show antibiotic resistance by preventing them in to the cell due to the mechanisms like decreased growth rate, expression of drug resistance genes and restricted penetration of antibiotic in to biofilms . ${ }^{1}$ Biofilm forming bacteria can combat antibiotic concentration 10-10000 folds higher than the essential concentration needed to hinder the growth of free floating bacteria. ${ }^{13}$

Out of a total of 347 Klebsiella pneumoniae isolated from our study, 167 isolates $(48.13 \%)$ were biofilm producers and $180(51.87 \%)$ biofilm non producers. The study conducted by Pramodhini et al showed $55.9 \%$ biofilm production among K.pneumoniae. ${ }^{9}$ Another study conducted by Thiyagarajan revealed $50 \%$ biofilm production among the strains of K.pneumoniae. ${ }^{20}$ Whereas the study conducted by Ruchi A Tayal et al suggested only $18 \%$ of biofilm production among the strains of K.pneumoniae. Biofilm producer exhibited the highest resistance to ceftazidime $(65.65 \%)$ followed by cefotaxime ((57.57\%), ceftriaxone $(56.56 \%)$, ciprofloxacin $(53.53 \%)$, gentamicin (32.32\%), amikacin (23.23\%), ertapenem (4.54\%) and meropenem $(2.02 \%)$. The non biofilm producers showed highest resistance to ceftazidime $(45.63 \%)$ followed by cefotaxime $((34.22 \%)$, ceftriaxone $(30.87 \%)$, ciprofloxacin (28.18\%), gentamicin (28.18\%), ertapenem (6.04\%) amikacin (5.36\%), and meropenem (3.14\%).Klebsiella pneumonia is one of the most important biofilm forming bacteria. This study showed the higher percentage of resistance towards various antibiotics predominantly among biofilm producers rather than non biofilm formers. Supporting our study Samia Beellifa et al observed that, the response of biofilm-growing strains showed higher incidence of antibiotic resistance than the non biofilm producing counterparts. ${ }^{12}$,A study conducted by Ruchi A Tayal et al showed the isolates of Klebsiella producing biofilm was multi drug resistantant and extend susceptibility to imipenem only. ${ }^{15}$ Mean while the results of present study suggested that, bio film formation reduces the efficacy of certain antimicrobial agents, whereas its effect is minimal on others. The findings were similar to those reported by other authors. ${ }^{8,10}$ Biofilms are more connected to ESBL producers than to non ESBL producers. ${ }^{4,6}$ Extended spectrum beta lactamase producing K.pneumoniae isolates had a greater ability to form biofilm than non biofilm producing isolates. ${ }^{2}$ Extended spectrum beta lactamases are plasmid mediated beta lactamases involved under the mechanism of drug resistance among the Enterobacteriaceae family due to the selective pressure imposed by extensive use of $3 \mathrm{rd}$ generation cephalosporins. ${ }^{9}$ Our study showed that, out of a total of 183 resistant strains of isolates of K.pneumoniae screened, 93(50.81\%) were ESBL producers and $70(75.26 \%)$ of 93 ESBL producers were biofilm producers. A study by Pramodhini et al showed $94.4 \%$ of ESBL strains of K.pneumoniae . ${ }^{9}$ In another study, Ariadnna CruzCórdova etal observed that, $100 \%$ of Klebsiella pneumoniae formed biofilm to varying degrees and ESBL positive strains of Klebsiella pneumoniae strains formed extensive biofilms. ${ }^{2}$ But the study done by Hemachandran et al showed $31 \%$ biofilm production among K.pneumoniae. ${ }^{16}$

\section{Conclusion}

The present study showed that there was high antibiotic resistance shown by K.pneumoniae for third generation cephalosporins. It also proved that, ESBL producing Klebsiella pneumoniae isolates had an exceptional potential to form biofilm in comparison with non ESBL forming K.pneumoniae.

\section{References}

[1] Afreenish Hasan, Javid Usman, Fatima Kaleem, Maria Omair, Ali Khalid, Muhammad Iqbal. Evaluation of different detection methods of biofilm formation in the clinical isolates. Braz J Infect Dis 2011;15:1413.

\section{Volume 5 Issue 2, February 2016}




\section{International Journal of Science and Research (IJSR) \\ ISSN (Online): 2319-7064 \\ Index Copernicus Value (2013): 6.14 | Impact Factor (2014): 5.611}

[2] Ariadnna Cruz-Córdova, Verónica Esteban-Kenel, Karina Espinosa-Mazariego, Sara A. Ochoa, Sarbelio Moreno Espinosa, Alicia de la Garza Elhain, Elizabeth Fernández Rendón, Edgar Oliver López Villegas, Juan Xicohtencatl-Cortes. Pathogenic determinants of clinical Klebsiella pneumoniae strains associated with their persistence in the hospital environment. Bol. Med. Hosp. Infant. Mex 2014;71:52-55.

[3] James H. Jorgensen, Mary Jane Ferraro. Antimicrobial Susceptibility Testing: A Review of General Principles and Contemporary Practices. Clinical Infectous Diseases 2009;49:1749-1755.

[4] Claudia Vuotto, Francesca Longo, Maria Pia Balice, Gianfranco Donelli, Pietro E. Varaldo.Antibiotic resistance related to biofilm formation in K.pneumoniae. Pathogens 2014;3:743-758.

[5] Dechen C Tsering, Shyamasree Das, Luna Adhiakari, Ranabir Pal, ${ }^{1}$ and Takhellambam SK Singh. Extended Spectrum Beta-lactamase Detection in Gram-negative Bacilli of Nosocomial Origin. J Glob Infect Dis. 2009 Jul-Dec; 1: 87-92.

[6] Lewis K. Riddle of biofilm resistance. Antimicrobial Agents Chemotherapy 2001;45:999- 1007.

[7] Merg-chuan wu, Tzu Forbes B.A., Sahm DF. Allice S, Weissfeld. Infections of urinary tract in Bailey \& Scott's diagnostic Microbiology, 11th Edition. St. Louis Missouri Eds,Mosby, USA 2002; 927-938.

[8] Mishra S K , Basukala P, Basukala O, Parajuli K, Pokhrel BM, Rijal B P. Detection of biofilm production and antibiotic resistance pattern in clinical isolates from indwelling medical devices. Curr Microbiol 2015 ;70:128-134.

[9] Pramodhini Subramaniaer, S Umadevi, Shailesh Kumar, Selvaraj Stephen. Determination of correlation between biofilm and ESBL producers of Enterobacteriaceae. Scholar's Research Journal 2012;2:2-6.

[10] Souli M, Giamarellou H. Effects of slime produced by clinical isolates of cooagulase negative Staphylococci on activities of various antimicrobial agents. Antimicrobial Agents Chemother 1998;42:939-941.

[11] Sanjay Chhibber, Deepika Nagand, Shruti Bansal. Inhibiting biofilm formation by Klebsiella Pneumoniae B5055 using an iron antagonizing molecule and a bacteriophage. BMC Microbiology 2013;13:174-81.

[12] Samia Bellifa, Hafida Hassaine, Damien Balestrino, Nicolas Charbonnel, ImanM hammeli, Ibtissem Kara Terki etal. Evaluation of biofilm formation of K.pneumoniae isolated from medical devices at the University hospital of Tlemeen, Algeria. AJMR 2013;7:5558-64.

[13] T Mathur, S Singhal, S Khan, D J Upadhyay, T Fatma, A Rattan. Detection of biofilm formation among the clinical isolates of Staphylococci:An evaluation of three different screening methods. Indian Journal of Medical Microbiology 2006;24:25-29.

[14] Adriana Marcia Nicolau Korres, Gloria Maria de Farias V. Aquije, David S. Buss, Jose Aires Ventura, Patricia Machado Bueno Fernandes, and Antonio Alberto Ribeiro Fernandes Comparison of Biofilm and Attachment Mechanisms of a Phytopathological and Clinical Isolate of Klebsiella pneumoniae Subsp. pneumonia2013;2013:1-6.
[15]Ruchi A Tayal, Sujata M Baveja, Anuradha S De. Analysis of biofilm formation and antibiotic susceptibility pattern of uropathogens in patients admitted in a tertiary care hospital.International Journal of Health and Allied Sciences 2015;4:247-252.

[16] Hemachandran. K., S. Bharathi, M. Radhakrishnan ,R. Balagurunathan. Studies on extended beta lactamase producing, biofilm forming clinical bacterial pathogens and its invitro inhibition by Actinobacterial extracts. Journal of Applied Pharmaceutical Science 2011; 1 :210-213.

[17] Bauer AN, Kirby WMM, Sherris J, etal. Antibiotic Susceptibility Testing by a Standardized Single disc Method. AMJ Clinical Pathol 1966; 45:493-496.

[18]Robert Schmieder; Robert Edwards. Insights into Antibiotic Resistance Through Metagenomic Approaches.Future Microbiol. 2012;7:73-89.

[19]Kenneth Todar. Bacterial resistance to antibiotics . Todar's Online Text book of Bacteriology page 3.

[20] Thiyagarajan Santhanamari, Jamal Alruwailiand Sathish Kumar. In vitroinhibition of ESBL positive Multidrug resistingUropathogenicbacteria using Coleus forskohlii. Int.J.Curr.Microbiol.App.Sci 2014;3: 431-444 